\section{Selective nuclear export of mRNAs is promoted by DRBD18 in} Trypanosoma brucei

Amartya Mishra ${ }^{1 *}$, Jan Naseer Kaur ${ }^{1 *}$, Daniel I. McSkimming ${ }^{2}$, Eva Hegedűsová ${ }^{3}$, Ashutosh P. Dubey $^{1}$, Martin Ciganda ${ }^{1}, Z_{\text {deněk Paris }}^{3,4}$, and Laurie K. Read ${ }^{1}$

${ }^{1}$ University at Buffalo, Jacobs School of Medicine and Biomedical Sciences, Buffalo, NY, USA; ${ }^{2}$ Bioinformatics and Computational Biology Core, University of Southern Florida, Tampa, FL, USA; ${ }^{3}$ Institute of Parasitology, Biology Centre, Czech Academy of Sciences, České Budějovice (Budweis), Czech Republic, ${ }^{4}$ Faculty of Science, University of South Bohemia České Budějovice (Budweis), Czech Republic.

Running title: DRBD18 controls mRNA export in Trypanosoma brucei

Keywords: Trypanosome, RNA binding protein, mRNA export, RNAseq, FISH, Nucleoporin

*equal contribution

To whom correspondence should be addressed:

Laurie K. Read

1read@,buffalo.edu

716-829-3307 


\section{SUMMARY}

2 Kinetoplastids, including Trypanosoma brucei, control gene expression primarily at the

3 posttranscriptional level. Nuclear mRNA export is an important, but understudied, step in this

4 process. The general heterodimeric export factors, Mex67/Mtr2, function in the export of mRNAs

5 and tRNAs in T. brucei, but RNA binding proteins (RBPs) that regulate export processes by

6 controlling the dynamics of Mex67/Mtr2 ribonucleoprotein formation or transport have not been

7 identified. Here, we report that DRBD18, an essential and abundant T. brucei RBP, associates with

8 Mex67/Mtr2 in vivo, likely through its direct interaction with Mtr2. DRBD18 downregulation

9 results in partial accumulation of poly $(\mathrm{A})^{+}$mRNA in the nucleus, but has no effect on localization

10 of intron-containing or mature tRNAs. Comprehensive analysis of transcriptomes from whole cell

11 and cytosol in DRBD18 knockdown parasites demonstrates that depletion of DRBD18 leads to

12 impairment of nuclear export of a subset of mRNAs. CLIP experiments reveal association of

13 DRBD18 with several of these mRNAs. Moreover, DRBD18 knockdown leads to a partial

14 accumulation of the Mex67/Mtr2 export receptors in the nucleus. Taken together, the current study

15 supports a model in which DRBD18 regulates the selective nuclear export of mRNAs by

16 promoting the mobilization of export competent mRNPs to the cytosol through the nuclear pore

17 complex. 


\section{INTRODUCTION}

2 Trypanosoma brucei species are early diverged unicellular eukaryotic parasites causing African

3 Sleeping Sickness in humans and Nagana in domestic animals (Keating et al., 2015, Büscher et

4 al., 2017). They are members of the family of Kinetoplastida, which includes other parasites such

5 as Trypanosoma cruzi, the causative agent of Chagas disease, and various species of Leishmania

6 that cause leishmaniases. These parasites all have complex life cycles that alternate between an

7 insect vector and the mammalian host. In T. brucei, two well studied life cycle stages are the

8 procyclic form ( $\mathrm{PF}$ ), which is found in the tsetse fly vector and the bloodstream form (BF), present

9 in the blood of the infected animal host (Matthews, 2005). T. brucei exhibits several unique

10 biological features, such as a condensed bipartite mitochondrial genome with its unique replication

11 machinery, uridine insertion/deletion RNA editing of mitochondrial mRNAs, as well as a complex

12 mechanism to evade the host immune system by frequent changes of variant surface glycoproteins

13 (Jensen \& Englund, 2012, Verner et al., 2015, Bangs, 2018, Zimmer et al., 2018, Aphasizheva et

14 al., 2020). The process of transcription in these parasites is also unusual. Most protein coding

15 genes are transcribed by RNA polymerase II to produce long polycistronic units which are co-

16 transcriptionally processed to monocistronic mRNAs. Individual mRNAs are produced by 5 ,

17 trans-splicing that adds a 39-nt long spliced leader RNA to the 5' end of each monocistronic RNA

18 and 3' addition of a poly(A) tail (Michaeli, 2011). Due to the absence of promoters upstream of

19 individual genes, gene regulation in T. brucei takes place primarily at the posttranscriptional level

20 (Clayton, 2019).

22 Posttranscriptional gene expression in eukaryotes involves multiple steps including processing of

23 nascent transcripts, nuclear export of mature mRNAs, and regulation of both mRNA decay and

24 translational efficiency. mRNA export machineries have been extensively studied in the model

25 organism Saccharomyces cerevisiae and later in metazoan species (Köhler \& Hurt, 2007,

26 Wickramasinghe \& Laskey, 2015). In the established universal model of mRNA export, pre-

27 mRNA is co-transcriptionally assembled to a messenger ribonucleoprotein (mRNP) by the TREX

28 complex, followed by mRNA processing and maturation. Adaptor proteins that exhibit RNA

29 binding activity recruit the heterodimeric export receptor Mex67/Mtr2 to the mRNP and promote

30 export through the nuclear pore complex (NPC) (Tutucci \& Stutz, 2011). In trypanosomes,

31 homologues of Mex67 and Mtr2 interact and function as nuclear export receptors for both mRNA 
1 and tRNA (Schwede et al., 2009, Dostalova et al., 2013, Hegedüsová et al., 2019). Nevertheless,

2 the domain organization of Mex67 in T. brucei is quite different than that of its yeast and metazoan

3 orthologues. Like yeast or metazoan Mex67, T. brucei Mex67 contains both leucine-rich repeat

4 (LRR) and an NTF2-like domains; however, it lacks an RNA recognition motif (RRM) and a

5 ubiquitin-associated domain (UBA) (Dostalova et al., 2013, Rink \& Williams, 2019). Moreover,

6 T. brucei Mex67 contains a $\mathrm{CCCH}$ zinc finger $(\mathrm{ZC} 3 \mathrm{H})$ motif in its N-terminal region, which is

7 essential for mRNA export (Dostalova et al., 2013). Other aspects of the nuclear export machinery

8 also differ in T. brucei compared to other well studied systems. Unlike yeast or metazoans,

9 trypanosomatids contain no recognizable components of the TREX complex, with the exception

10 of the DEAD box helicase, Sub2 (Serpeloni et al., 2011a, Dostalova et al., 2013). Recent studies

11 suggest that active transcription is necessary to initiate mRNA export in T. brucei, although neither

12 the completion of transcription nor splicing appear to be essential for export (Goos et al., 2018).

14 RNA binding proteins (RBPs) play major roles in gene expression and development in

15 trypanosomes. In some cases, RBPs have been shown to bind specific cis-acting regulatory

16 elements present in the 3'-untranslated regions (3'-UTRs) of mRNAs to modulate mRNA stability

17 or translation (Kolev et al., 2014, Clayton, 2019). RBPs are classified into different groups based

18 on the presence of distinct RNA binding domains, with RNA recognition motif(RRM) and ZC3H-

19 containing proteins being the most common RBPs in trypanosomes (Kolev et al., 2014). Examples

20 of the impacts of RBPs in T. brucei include the critical roles in developmental gene regulation

21 played by RRM-containing RBPs, RBP10 and RBP6, and REG9.1 (Kolev et al., 2012, Mugo \&

22 Clayton, 2017, Rico et al., 2017). With regard to ZC3H proteins, ZC3H11 mediates gene

23 expression during heat shock (Droll et al., 2013), and ZC3H20 binds specifically to a subset of

24 mRNAs expressed in PFs (Liu et al., 2020).

DRBD18 is an essential and abundant an RRM-containing RBP in T. brucei (Lott et al., 2015).

27 Our previous studies demonstrated that depletion of DRBD18 causes extensive transcriptome 28 rearrangement in PF T. brucei through both stabilization or destabilization of distinct subsets of 29 mRNAs. The molecular functions of DRBD18 in mRNA stabilization or destabilization and 30 protein-protein interactions are markedly controlled by methylation of arginine residues present 31 between its two RRM domains. Towards an understanding of the biochemical mechanisms of 
1 DRBD18 function, mass spectrometry analysis of Tandem Affinity Purified (TAP)-tagged 2 DRBD18 revealed the interaction of DRBD18 with numerous proteins involved in RNA biology, 3 including the general mRNA export receptors Mex67 and Mtr2, and the Sub2 RNA helicase (Lott 4 et al., 2015). Previous immunofluorescence analysis revealed a strong perinuclear concentration 5 of DRBD18 that, together with its interaction with Mex67/Mtr2, is consistent with a role for DRBD18 in nucleocytosolic mRNA transport (Lott et al., 2015). In the present study, we confirm the in vivo association between DRBD18 and Mex67/Mtr2 and demonstrate that DRBD18 and Mtr2 directly interact in vitro. High throughput sequencing and immunofluorescence studies show that DRBD18 promotes the export of a subset of mRNAs from nucleus to cytosol. At the same time, DRBD18 also facilitates the export of a fraction of Mex67/Mtr2 from the nucleus. In contrast, DRBD18 does not share a function with Mex67/Mtr2 in the export of intron-containing or mature tRNAs. The mRNA export machinery is not well characterized in T. brucei due to the absence of many conserved export factors. The current study is the first to document a T. brucei RBP that

\section{RESULTS}

\section{$17 \quad 2.1$ DRBD18 interacts with export receptor proteins Mex67 and Mtr2 in vivo}

18 Our previous mass spectrometry analysis suggested that DRBD18 associates with several RNA 19 processing complexes, including the nuclear export factors, Mex67 and Mtr2 (Lott et al., 2015).

20 These two factors are evolutionarily conserved and typically form a heterodimer, although distinct

21 functions for Mex67 and Mtr2 have also been reported (Braun et al., 2002, Dostalova et al., 2013,

22 Hegedüsová et al., 2019). To explore whether DRBD18 plays a role in Mex67/Mtr2 mediated 23 RNA export, we began by confirming the interactions of Mex67 and Mtr2 with DRBD18 in vivo.

24 For this purpose, we generated PF T. brucei cells expressing HA-tagged Mtr2 from its genomic

25 locus. Immunoprecipitation (IP) using anti-HA antibody in the aforementioned cell line confirmed 26 that Mtr2 associates with DRBD18, as well as with Mex67 as expected, while both are absent in a 27 control IP (Fig. 1A). As DRBD18 is an RNA binding protein (Lott et al., 2015), we next asked 28 whether the DRBD18-Mtr2 interaction is impacted by RNA by performing co-IPs using HA-Mtr2 29 tagged T. brucei cell lysate treated either with an RNase cocktail or with RNase inhibitor (Figs. 30 1B and 1C). DRBD18 consistently displayed an increased interaction with Mtr2 in the absence of 31 RNA, whereas the interaction between Mex67 and Mtr2 was unaffected by RNA. To further probe 
1 the RNA dependence of DRBD18-Mex67/Mtr2 interaction, we immunoprecipitated DRBD18 2 with anti-DRBD18 antibody from the HA-Mtr2 tagged T. brucei cell lysate in presence and 3 absence of RNase, and demonstrated increased co-immunoprecipitation of both Mex67 and Mtr2 4 with DRBD18 in RNase treated samples (Figs. 1D and 1E). Thus, we conclude that DRBD18 5 associates with Mex67/Mtr2 in vivo, and that this interaction is strengthened in the absence of 6 RNA.

\subsection{DRBD18 directly interacts with Mtr2 in vitro}

Having demonstrated the in vivo DRBD18-Mex67/Mtr2 interaction, we next asked if DRBD18 interacts directly with the export receptors. To this end, we expressed and purified recombinant

11 His-Mtr2, GST-DRBD18, and GST in E. coli as described in Experimental Procedures. Purity of 12 the recombinant proteins was analyzed by SDS-PAGE analysis (Fig. 2A). Next, we performed 13 GST-pulldown assays using GST-DRBD18 as bait protein, GST as a negative control, and purified 14 His-Mtr2 as prey protein. For this purpose, we incubated equimolar amounts of purified His-tagged 15 Mtr2 with the immobilized GST or GST-DRBD18. Western blot analysis showed that Mtr2 is 16 pulled down by the GST-tagged DRBD18 but not by GST, indicating that DRBD18 interacts 17 directly with Mtr2 (Fig. 2B). These data collectively demonstrate that DRBD18 interacts with 18 Mtr2 through a protein-protein interaction.

\subsection{DRBD18 does not impact Mex67/Mtr2-dependent export of tRNAs}

21 To begin to understand the functional significance of the DRBD18-Mex67/Mtr2 interaction, we

22 first confirmed that DRBD18 does not alter the steady state levels or heterodimerization of Mex67 23 and Mtr2 (Fig. S1). As these parameters are unaffected by DRBD18 knockdown, the interaction 24 between DRBD18 and Mex67/Mtr2 suggests that DRBD18 could impact some functions of these 25 export factors. It was recently reported that Mex67 and Mtr2 play important and distinct roles in 26 tRNA nuclear export in T. brucei (Hegedüsová et al., 2019). Knockdown of Mtr2 results in nuclear 27 accumulation of all type of tRNAs, while Mex67 knockdown leads to nuclear accumulation of 28 subsets of tRNAs that are modified with queuosine. To investigate the functional role of the 29 DRBD18-Mex67/Mtr2 interaction in tRNA export, we analyzed total RNA from uninduced and 30 induced DRBD18 RNAi cells by Northern hybridization. We first monitored the splicing of 31 tRNA ${ }^{\text {Tyr }}$, which occurs exclusively in the cytosol in T. brucei and, thus, serves as a measure of the 
1 export of immature, intron-containing tRNA ${ }^{\text {Tyr }}$. We observed no effect of DRBD18 knockdown

2 on this parameter, similar to the lack of Mex67 or Mtr2 impact on export of this immature tRNA

3 (Fig. S2). Following splicing, tRNA ${ }^{\text {Tyr }}$ undergoes retrograde import into the nucleus, at which

4 point it acquires a queuosine modification and is then re-exported. Impairment of this re-export

5 step, which requires both Mex67 and Mtr2, results in aberrantly high queuosine levels on tRNA ${ }^{\text {Tyr }}$

6 (Hegedüsová et al., 2019). We next asked whether DRBD18 exerts any modulation of the function

7 of Mex67 and Mtr2 in the re-export of tRNA ${ }^{\text {Tyr }}$ by analyzing the level of queuosine modified

8 tRNA $^{\text {Tyr }}$ using boronate affinity gel electrophoresis followed by Northern blot hybridization

9 (Kessler et al., 2018). RNA samples treated with the oxidizing agent, sodium periodate, served as

10 a negative control (Fig. 3A). We detected similar levels of queuosine modified tRNA ${ }^{\text {Tyr }}$ in RNA

11 from uninduced and induced DRBD18 RNAi cells (Fig. 3A), indicating that DRBD18 does not

12 impact the Mex67/Mtr2 mediated re-export of queuosine modified tRNA ${ }^{\text {Tyr }}$. To further investigate

13 the potential role of DRBD18 in tRNA export, we analyzed mature tRNA ${ }^{\text {Tyr }}$ and tRNA ${ }^{\text {Glu }}$ (Figs.

$143 \mathrm{~B}$ and $3 \mathrm{C}$ ) by fluorescence in situ hybridization (FISH) using specific fluorophore labeled

15 oligonucleotide probes (Table S1). These probes detect spliced tRNA, regardless of its queuosine

16 modification status. Unlike what is observed in Mtr2 or Mex67 knockdowns, we did not observe

17 any nuclear accumulation of mature tRNA ${ }^{\text {Tyr }}$ or tRNA ${ }^{\text {Glu }}$ when DRBD18 is knocked down.

18 Collectively, these data show that DRBD18 does not impact the functions of Mex67 or Mtr2 with

19 respect to their roles in the export or re-export of tRNAs.

\subsection{DRBD18 plays role in $\mathrm{mRNA}$ export from nucleus to cytosol}

22 Mex67/Mtr2 plays an important role in mRNA transport from nucleus to cytosol in T. brucei 23 (Dostalova et al., 2013), but the mechanisms of export are poorly understood. The interaction of 24 DRBD18 with these export factors (Figs. 1 and 2) suggests that DRBD18 could modulate export 25 of at least some mRNAs. To determine whether DRBD18 exerts any functional role on mRNA 26 export from nucleus to cytosol, we first assessed the subcellular location of poly(A) ${ }^{+}$RNA in 27 DRBD18 RNAi uninduced and induced cells by oligo(dT) FISH (Fig. 4A). To quantify the mRNA 28 distribution in the nucleus, we plotted the fluorescence intensities of the FISH and DAPI signals 29 across the nucleus, with overhangs covering the cytosol, across multiple cells (Fig. 4B). In 30 DRBD18 RNAi induced cells, we observed substantial overlap between FISH signals and DAPI 31 signals (lower panel), while uninduced cells exhibited minimum overlap between two fluorophores 
1 (upper panel). These observations demonstrate that DRBD18 depletion causes partial 2 accumulation of mRNA in nucleus, thereby indicating a role for DRBD18 in export of mature mRNA from nucleus to cytosol.

\subsection{DRBD18 regulates gene expression by promoting the export of a subset of $m R N A s$}

The partial nuclear retention of mRNA in DRBD18 depleted cells (Fig. 4) suggests that DRBD18 impacts nuclear export of only a subset of mRNAs. To identify a cohort of mRNAs whose export is affected by DRBD18, we carried out Illumina NextSeq analysis of total RNA and cytosolic RNA from uninduced and induced DRBD18 RNAi cells. We identified mRNAs whose abundance is unchanged in whole cell RNA, but decreased in cytosolic RNA upon DRBD18 RNAi, as an indication of decreased nuclear export (Müller-McNicoll et al., 2016, Rehwinkel et al., 2004, Wickramasinghe et al., 2014). The purity of subcellular fractionation and the depletion of DRBD18 by RNAi induction were confirmed by Western blot analysis using antibodies against cytosolic and nuclear marker proteins and anti-DRBD18 antibody, respectively (Fig. S3). In whole cell RNA, we identified 259 transcripts that decreased in abundance and 378 that increased upon DRBD18 knockdown (1.5 fold change; corrected p-value <0.05) (Table S2), similar to our previously published findings (Lott et al., 2015). In the cytosolic fraction, we identified 216 transcripts with decreased abundance and 593 transcripts with increased abundance (1.5 fold change; corrected p-value $<0.05$ ) upon DRBD18 knockdown (Table S3, Fig. S4). Next, we focused on the set of transcripts whose nuclear export was apparently promoted by DRBD18, consistent with the partial nuclear accumulation of poly $(\mathrm{A})^{+}$RNA that we observed by FISH (Fig. 5). To this end, we identified mRNAs reduced in cytosol and unchanged in the whole cell upon DRBD18 RNAi (Fig. 5A; Fig. S4). Of the 174 transcripts fitting these parameters (corrected p24 value $<0.05$; Fig. 5A), 95 exhibited a fold decrease in the cytosol of $>1.5$ fold (Fig. 5A, blue).

25 These 95 transcripts comprise the list of potential DRBD18 facilitated export targets (Table S4). No significantly enriched GO terms or nucleotide motifs were apparent in this mRNA set. To 27 validate the relative expression of the transcripts in cytosol and whole cell in DRBD18 RNAi 28 induced or uninduced cells, we performed qRT-PCR analyses on a subset of these transcripts. By 29 qRT-PCR, we detected either no decrease or small decreases in the transcripts in whole cell RNA 30 samples as expected (Figs. 5B and S5), and we confirmed significantly larger decreases in the 31 cytosol for seven of the transcripts tested (Fig. 5B). An additional four transcripts showed no 
1 significant differences between whole cell and cytosol, although some appeared to trend in this

2 direction (Fig. S5). If the nuclear export of these transcripts is directly promoted by DRBD18,

3 we would expect them to associate with DRBD18 in vivo. To test this, we performed cross-linking

4 immunoprecipitation (CLIP) experiments in which we measured the enrichment of the transcripts

5 in Fig. 5B using anti-DRBD18 antibodies relative to a non-specific antibody control. Indeed, all

6 seven transcripts were enriched, from 2- to 17-fold, while a negative control transcript

7 (Tb927.7.7400; Table S2) was not (Fig. 5C). The reduced abundance of specific mRNAs in cytosol

8 compared to that in the whole cell RNA population upon DRBD18 depletion is consistent with a

9 function for DRBD18 in the export of these mRNAs. Moreover, DRBD18 binds differentially

10 transported mRNAs in vivo, suggesting it plays a direct role in promoting the nucleocytosolic

11 transport of a subset of mRNAs.

$13 \quad 2.7$ DRBD18 levels impact the relative abundance of export receptors in nucleus and cytosol

$14 m \boldsymbol{R} N \boldsymbol{A}$

15 One mechanism by which DRBD18 may promote export of selected mRNAs is by facilitating the

16 nuclear export of the Mex67/Mtr2-DRBD18 mRNP. To determine whether DRBD18 impacts the

17 transport of Mex67/Mtr2 from nucleus to cytosol, we fractionated DRBD18 RNAi induced and

18 uninduced cells into nuclear and cytosolic fractions, and validated the fractionation by western

19 blot analysis of histone $\mathrm{H} 3$ and $\mathrm{EF} 1 \alpha$, respectively (Figs. 6A and 6B). We then blotted for Mex67

20 and Mtr2 in both fractions and normalized their expression to the expression of histone H3

21 (nucleus) or EF1 $\alpha$ (cytosol). We detected a significant and reproducible approximately two-fold

22 increase in nuclear Mex67 and Mtr2 in cells depleted of DRBD18. We did not detect significant

23 concomitant changes in Mtr2 or Mex67 level in cytosolic fraction of DRBD18 RNAi induced and

24 uninduced cells, possibly due to the greater abundance of Mex67/Mtr2 in the cytosol in our assay

25 system. These data indicate that DRBD18 modestly impacts the dynamics of export receptor

26 trafficking between the two subcellular compartments, consistent with its ability to promote the

27 export of a subset of mRNAs.

\section{DISCUSSION}

30 Gene expression in kinetoplastids, including T. brucei, is modulated primarily at the 31 posttranscriptional level, with RBPs constituting the key gene regulatory factors (Clayton, 2019). 
1 While RBPs and cis-acting sequences that modulate mRNA stability and translation have been

2 identified in T. brucei, no proteins outside the basal nuclear export machinery have been reported

3 to control mRNA export (Schwede et al., 2009, Dostalova et al., 2013). Here, we show that the

4 abundant and essential T. brucei RBP, DRBD18, promotes the nucleocytosolic transport of a

5 subset of mRNAs. While nuclear mRNA export was originally thought to be constitutive, more recent studies reveal that nuclear export of mRNAs can be highly selective, and this process is now established as an important level of gene regulation (Wickramasinghe \& Laskey, 2015). In

8 mammals, processes such as DNA repair, maintenance of pluripotency, stress responses, and cell proliferation can be regulated by selective nuclear export promoted by distinct proteins. We demonstrate here that T. brucei DRBD18 binds the general nuclear export receptor, Mex67/Mtr2,

11 likely through a direct interaction with Mtr2, and facilitates the export of a subset of mRNAs.

12 Thus, DRBD18 is the first reported nuclear mRNA export specificity factor in trypanosomes.

Bulk nuclear mRNA export is facilitated in yeast and mammals by the TREX complex,

16 helicase, homologues of TREX complex components are absent in T. brucei (Serpeloni et al.,

17 2011b); however, DRBD18 clearly does not fulfill the bulk export role of a TREX component, as

18 it impacts the export of only a few hundred mRNAs. Indeed, it has been suggested that T. brucei

19 Mex67 may not utilize a homolog of the TREX complex to increase bulk mRNA binding due to

20 the presence of a kinetoplastid-specific ZC3H domain at its $\mathrm{N}$ terminus (Dostalova et al., 2013).

21 DRBD18 may act as an adaptor protein that promotes association of a select subset of mRNAs

22 with Mex67/Mtr2. Alternatively, or in addition, DRBD18 may function as a nuclear export

23 chaperone. The nuclear envelope defines the physical barrier between nucleoplasm and cytosol,

24 and communication between the two compartments is mediated by cylindrical macromolecular

25 NPCs. Rate limiting steps in mRNA nuclear export are diffusion of mRNA loaded Mex67/Mtr2

26 through the nucleoplasm and loading onto NPC, the latter of which requires the activity of

27 chaperones that direct cargo to the NPC (Scott et al., 2019). Chaperoning of Mex67/Mtr2 to the

28 NPC has been proposed as a good target for regulation of the export rate of specific subsets of 29 mRNAs (Scott et al., 2019). 
One clue as to how DRBD18 might facilitate export of mRNAs bound by the Mex67/Mtr2DRBD18 mRNP lies in the reported interactions of Mex67 and DRBD18 with NPC components.

The NPC consist of multiple copies of $\sim 30$ proteins termed nucleoporins (Nups), of which $T$.

4 brucei contains two major classes: phenylalanine-glycine (FG) repeats Nups and core scaffold

5 Nups (Fig. 7) (Obado et al., 2016). The core scaffold, which is comprised of two inner ring and two outer ring structural components interacts with the nuclear envelope. FG Nups are disordered proteins responsible for the selective permeability of the NPC to nucleocytosolic transport, playing vital roles in the transport of the soluble transport receptors through hydrophobic interactions (Strambio-De-Castillia et al., 2010). Comprehensive proteomic and interactome analysis of $T$. brucei NPC by Obado, et al. (Obado et al., 2016) revealed both similarities and differences in the NPC protein composition in this excavate compared to that of the opisthokonts (humans and yeast). Further, affinity capture of T. brucei Mex67 revealed its association with many components of the NPC (Obado et al., 2016). Under high stringency, Mex67 interactions were observed predominantly with the less evolutionarily conserved outer ring and FG Nups. In particular, the retention of FG Nups comprising the Nup76 complex (Nups 76, 140, and 149; see Fig. 7) under high stringency implicates the Nup76 complex as part of the mRNA export docking platform.

17 Previous Tandem Affinity Purification (TAP) of DRBD18-TAP from PF T. brucei also returned several NPC components (Lott et al., 2015). Remarkably, these studies identified as DRBD18 interacting NPC proteins almost exclusively those that also showed high stringency interactions with Mex67, including components of the Nup76 complex (Nups140 and 149) and the outer ring (Nups 158, 132, 109, 89, and 82). Interaction of DRBD18 with the Nup76 complex docking platform may increase the frequency and/or affinity with which the Mex67/Mtr2 complex interacts with the NPC, thereby facilitating export of mRNAs bound to the Mex67/Mtr2-DRBD18 complex

24 (Tetenbaum-Novatt \& Rout, 2010). Future studies will be needed to address the precise mechanism by which DRBD18 promotes the export of a distinct mRNA subset.

While DRBD18 clearly facilitates the export of a subset of mRNAs as described here, we also identified numerous mRNAs whose export may be inhibited by DRBD18. Over 500 mRNAs are increased in the cytosol upon DRBD18 knockdown, and approximately 200 of these mRNAs 30 are not increased in whole cell RNA pools (Fig. S4). Thus, it is likely that DRBD18 has dual and 31 opposing roles in mRNA nuclear export. In model organisms, posttranslational modifications of 
1 the nuclear export machinery impact numerous steps in this process (Tutucci \& Stutz, 2011, 2 Howard \& Sanford, 2015, Wickramasinghe \& Laskey, 2015). One potential mechanism for 3 regulation of disparate functions of DRBD18 in mRNA export in T. brucei is the methylation of 4 arginine residues that lie between the two DRBD18 RRM domains (Lott et al., 2015). We 5 previously demonstrated that arginine methylation promotes the ability of DRBD18 to stabilize mRNAs, while it inhibits the DRBD18 mediated destabilization of other transcripts. With regard to Mex67/Mtr2, DRBD18 TAP returned increased numbers of Mex67 peptides with 8 hypomethylated DRBD18, but increased Mtr2 peptides with methylmimic DRBD18, so the impact of methylation on the DRBD18-Mex67/Mtr2 interaction is unresolved. Additionally, with a few exceptions, most Nups appeared to bind equivalently to hypomethylated and methylmimic

11 DRBD18 (Lott et al., 2015). It has been suggested that selective mRNA nuclear export in mammals 12 is controlled by combinatorial binding of distinct RBP combinations to a given transcript 13 (Wickramasinghe \& Laskey, 2015). Interestingly, TAP of hypomethylated and methylmimic 14 DRBD18 identified several RRM and ZC3H RBPs that were differentially bound to DRBD18 in 15 RNase-treated extracts depending on its methylation status (Lott et al., 2015). This finding 16 suggests that these DRBD18 associated RBPs could contribute to a combinatorial effect on nuclear 17 mRNA export in T. brucei with distinct impacts based on the methylation status of DRBD18.

Nuclear mRNA export interfaces with other RNA processing and gene regulatory events (Howard \& Sanford, 2015, Wickramasinghe \& Laskey, 2015). One such process is nuclear mRNA 21 decay. Nuclear retained mRNAs are generally turned over, and the balance between mRNA export 22 kinetics and decay can be a key determinant of gene expression (Meola \& Jensen, 2017, Tudek et $a l ., 2019)$. In yeast, experimental inhibition of nuclear export leads to the degradation of protein 24 coding transcripts (Tudek et al., 2018). In light of these findings, our approach to identifying transcripts whose nuclear export is promoted by DRBD18 may have been conservative. Because we identified only those mRNAs whose abundance was statistically unchanged in the whole cell

27 RNA pool, but decreased in cytosol, we would not have identified those mRNAs whose abundance 28 was significantly decreased by decay upon nuclear retention. Thus, the impact of DRBD18 in 29 promoting mRNA export may be broader than reported here. Nuclear mRNA export can also be 30 coupled to transcript specific translation by nucleocytosolic shuttling proteins, such as some SR 31 proteins (Maslon et al., 2014, Howard \& Sanford, 2015, Brugiolo et al., 2017). DRBD18 localizes 
1 to both nucleus and cytosol, with a perinuclear concentration (Fig. S3) (Lott et al., 2015). This

2 localization pattern, together with its interaction with numerous NPC components, suggests that

3 DRBD18 may undergo nucleocytosolic shuttling. Interestingly, DRBD18-TAP purification

4 revealed the protein's interaction with ribosomes and translation initiation factors (Lott et al.,

5 2015). In addition, Klein, et al. reported association of DRBD18 with bloodstream form T. brucei

6 polysomes (Klein et al., 2015). Thus, one possible scenario entails sequence specific coupling of

7 mRNA nuclear export and translation by DRBD18 to enhance gene expression. Together, our data

8 suggest that DRBD18 is a multifunctional gene regulatory protein with the potential to integrate mRNA export, stability, and translation.

\section{EXPERIMENTAL PROCEDURES}

\subsection{Cell line generation and culture}

14 PF T. brucei strain 29-13 and all cell lines derived from this strain were grown at $27^{\circ} \mathrm{C}$ in $\mathrm{SM}$ 15 medium supplemented with $10 \%$ fetal bovine serum and containing hygromycin $\left(50 \mu \mathrm{g} \mathrm{ml}^{-1}\right)$ and 16 G418 $\left(15 \mu \mathrm{g} \mathrm{m}^{-1}\right)$. Cells harboring the doxycycline inducible DRBD18 (Tb927.11.14090) RNA 17 interference (RNAi) constructs described previously in (Lott et al., 2015) were grown with the 18 addition of phleomycin $\left(2.5 \mu \mathrm{g} \mathrm{ml}^{-1}\right)$. To induce RNAi, the clonal cell line was incubated for $19 \mathrm{~h}$ 19 (high throughput sequencing and qRT-PCR) or $23 \mathrm{~h}$ (all other experiments) with $4 \mu \mathrm{g} \mathrm{m} \mathrm{m}^{-1}$ 20 doxycycline. In situ tagging of TbMtr 2 with a triple hemagglutinin (3XHA) tag was performed by

21 the long primer PCR method, using the pPOTv6 plasmid according to the published protocol by 22 (Dean et al., 2015). Primers are listed in Table S1. PCR products were transfected into T. brucei 23 for genomic integration, and transfectants were selected with blasticidin $\left(20 \mu \mathrm{g} \mathrm{ml}^{-1}\right)$.

\subsection{Immunoprecipitation and Western blot}

26 Approximately $1.5 \times 10^{9}$ cells were collected, washed with PBS, and resuspended in lysis buffer 27 (50 mM HEPES (pH 7.4), 3 mM MgCl $2,150 \mathrm{mM} \mathrm{KCl,} 150$ mM sucrose, 0.5\% Tween-20, EDTA28 free protease inhibitor cocktail (Roche, USA), and 1mM DTT). Cells were lysed by sonication 29 (Sonic Dismembrator, Fisher Scientific, USA) three times for $15 \mathrm{~s}$ at $30 \mathrm{~s}$ intervals on ice at $60 \%$ 30 amplitude, and clarified by centrifugation at $15,000 \mathrm{xg}$ for $30 \mathrm{~min}$ at $4^{\circ} \mathrm{C}$. For immunoprecipitation 31 with anti-HA antibody, clarified lysates were incubated with agarose immobilized rabbit anti-HA 32 beads (ICL Laboratories, USA) for $2 \mathrm{~h}$ at $4{ }^{\circ} \mathrm{C}$. As a negative control, lysates were incubated with 
1 IgG Sepharose beads (GE Healthcare, USA) for $2 \mathrm{~h}$ at $4^{\circ} \mathrm{C}$. Bound proteins were eluted with 100 $2 \mathrm{mM}$ glycine $(\mathrm{pH} 2.5)$ and the solution neutralized using $1 \mathrm{M}$ Tris buffer $(\mathrm{pH}$ 7.5). Proteins were 3 electrophoresed on a 12.5\% SDS-PAGE gel and transferred to nitrocellulose membrane. 4 Membranes were blocked with 5\% non-fat dry milk in Tris buffered saline with Tween-20 (TBST) 5 and probed with polyclonal antibodies against DRBD18 (Lott et al., 2015), p22 (Hayman et al., 2001) and Mex67 (a kind gift from Prof. Mark Carrington, University of Cambridge, UK). Commercially available antibodies against HA-epitope (Mouse monoclonal, Thermo Fisher Scientific), Histone H3 (Rabbit polyclonal, Abcam), and EF1 $\alpha$ (Mouse monoclonal, Santa Cruz Biotechnology) were also used. Blots were washed with TBST and subsequently probed with secondary antibodies, either goat anti-rabbit HRP or goat anti-mouse HRP. Signals were detected using an ECL preparation as recommended by the manufacturer (Thermo Fisher Scientific, USA), 13 software.

\subsection{Protein expression and GST pulldown assay}

16 Expression of recombinant GST-tagged DRBD18 was carried out as described earlier (Lott et al., 17 2015). For His-Mtr2 (Tb927.7.5760), the ORF was cloned into the MCS-1 of pETDuet-1vector 18 (with MSC2 left empty), with frame shift correction by insertion of single nucleotide before the 19 start codon (Kafková et al., 2017) and was purified using immobilized metal affinity 20 chromatography (IMAC) as described earlier (Klebanov-Akopyan et al., 2018). For GST pulldown 21 assays, all recombinant plasmids including pGEX4T-1, were transformed into Escherichia 22 coli BL21 strain, and protein expression was induced by addition of $0.5 \mathrm{mM}$ isopropyl 1-thio-D23 galactopyranoside (IPTG) and subsequent growth at $22^{\circ} \mathrm{C}$ overnight. Cells expressing GSTDRBD18 or GST were harvested and resuspended in lysis buffer (50 mM Tris- $\mathrm{HCl}(\mathrm{pH} \mathrm{8.0),} 250$ $\mathrm{mM} \mathrm{NaCl}, 5 \mathrm{mM}$ EDTA, $10 \mathrm{mM}$ DTT, and 1mM PMSF) and lysed by four cycles of maximumpower sonication bursts of $30 \mathrm{~s}$ each at $4^{\circ} \mathrm{C}$. Triton X-100 was added to a final concentration of $1 \%$, followed by a 30 min centrifugation at $15,000 \mathrm{xg}$ and $4^{\circ} \mathrm{C}$. The supernatant was incubated with Glutathione-Sepharose 4B beads (GE) at $4^{\circ} \mathrm{C}$ for $2 \mathrm{~h}$. The column was extensively washed with ice cold lysis buffer. Equal amounts of purified His-Mtr2 were added and incubated for another $2 \mathrm{~h}$ at $4^{\circ} \mathrm{C}$ with the immobilized GST or GST fusion protein. The columns were washed with ice cold wash buffer. The beads were boiled with SDS-PAGE Laemmli buffer 
and analyzed by Western blot using anti-Histidine (anti-Mouse, Sigma Aldrich) and anti-GST antibodies (anti-Rabbit, Sigma Aldrich).

\subsection{RNA isolation from nuclear-cytosolic fractionation}

5 Nuclear-cytosolic fractionation was performed in triplicate as described previously in Dostalova, et al. (Dostalova et al., 2013). Briefly, $2.5 \times 10^{8} \mathrm{PF}$ cells were harvested, washed in phosphate buffer saline (PBS), and resuspended in PBS. Nonidet-P40 was added to final concentration of $0.1 \%$, the cell suspension was mixed thoroughly, and nuclei were pelleted by centrifugation at 2300xg for $1 \mathrm{~min}$. Supernatant was considered as cytosolic fraction. To validate the fractionation and DRBD18 knockdown, samples corresponding to same cell equivalents from whole cells and supernatant (cytosolic) fractions were subjected to SDS-PAGE and Western blot analysis with anti-DRBD18, anti-histone H3, and anti-EF1 $\alpha$ antibodies. For RNA isolation, Trizol reagent (Invitrogen) was added to the cytosolic fraction and this suspension frozen at $-80^{\circ} \mathrm{C}$. Total RNA was isolated from whole cell and cytosolic fractions using Trizol reagent and phenol chloroform extraction followed by DNase-I treatment (DNA-free DNase kit; Ambion). The RNA was further purified using phenol chloroform extraction. Purified RNA samples were submitted for RNA

\subsection{RNA library preparation, sequencing, and bioinformatic analysis}

20 Prior to library preparation, total RNA was quality checked using an Agilent Fragment Analyzer

21 to assess quality and Qubit Fluorescence (Invitrogen) to measure concentration. RNA libraries 22 were prepared following the New England Biolabs NEXT UltraII Directional RNA library kit 23 using polyA + magnetic beads. Following library preparation, concentration and quality control, 24 final libraries were pooled to $10 \mathrm{nM}$ and the concentration of the pool was determined using the 25 Kapa Biosystems Universal qPCR kit. After dilution and denaturing, the pooled library was loaded 26 onto a NextSeq 500 high output flow cell (PE75) for sequencing. Sequencing quality was assessed using FastQC and MultiQC, and low-quality bases were removed using TrimGalore, a cutadapt wrapper (Andrews, 2010, Krueger, 2015, Ewels et al., 2016, Martin, 2011). Ribosomal RNA

29 (rRNA) reads were removed with SortMeRNA prior to alignment with Spliced Transcripts 30 Alignment to a Reference (STAR) against the TREU927 T. brucei reference genome, downloaded 31 from TriTrypDB v41 (Kopylova et al., 2012, Dobin et al., 2013, Aslett et al., 2010). Transcript 
1 abundances were estimated with RNA-Seq by Expectation Maximization (RSEM) (Li \& Dewey,

2 2011). Differential abundances were identified using differential expression analysis for sequence

3 count data (DESeq2) (Love et al., 2014).

5 The sequencing data used in this study has been deposited in Sequence Read Archive, accession number GSE158584.

\section{6 qRT-PCR analysis}

Reverse transcription (RT) was performed with $1 \mu \mathrm{g}$ of DNase I-treated RNA following standard procedures with random hexamer primers and iScript reverse transcriptase (BioRad). Quantitative

11 reverse transcription PCR (qRT-PCR) reactions were performed using primer pairs targeted at 12 specific transcripts (Table S1). Amplification was performed using a CFX Connect Real Time

13 System (Bio Rad), and data were analyzed using BioRad CFX Manager 3.1. Results were analyzed 14 using the Bio-Rad CFX Manager 3.1 software. RNA levels were normalized to 18S rRNA using 15 the standard curve method.

\section{$17 \quad 4.7$ Fluorescence in situ hybridization (FISH)}

18 For tRNA FISH analysis, cells were harvested, fixed and permeabilized as described (Hegedüsová et al., 2019) and then pre-hybridized for $2 \mathrm{~h}$ with hybridization solution (2\% BSA, 5X Denhardt's

20 solution, $4 \mathrm{X} \mathrm{SSC}$, 5\% dextran sulphate, 35\% deionized formamide, $10 \mathrm{U} \mathrm{ml}^{-1}$ RNase inhibitor).

21 The slides were then incubated overnight at room temperature in a humid chamber in the presence

22 of $10 \mathrm{ng}^{-1} \mathrm{l}^{-1}$ fluorophore labeled oligonucleotide probes (Supplementary Table S1), in the 23 hybridization solution. Slides were washed as described earlier (Hegedüsová et al., 2019) and 24 mounted with mounting medium supplemented DAPI. Images were taken with confocal 25 microscope Olympus Fluo ViewTM FV1000.

27 mRNA FISH analysis was performed as previously described (Dostalova et al., 2013). Briefly, 2.5

28 X $10^{7}$ cells were harvested by centrifugation, washed, resuspended in PBS, and allowed to adhere 29 to poly-L-lysine coated slides for $30 \mathrm{~min}$. Cells were fixed with $4 \%$ paraformaldehyde followed 30 by a 10 min treatment with $25 \mathrm{mM}$ ammonium chloride. Fixed cells were permeabilized for $1 \mathrm{~h}$ in 31 blocking buffer (PBS containing 0.5\% saponin, 2\% BSA and 10U ml-1 RNase inhibitor). 
1 Permeable cells were pre-hybridized for $2 \mathrm{~h}$ with hybridization solution (2\% BSA, 5X Denhardt's 2 solution, 4X SSC, 5\% dextran sulphate, 35\% formamide, $0.5 \mu \mathrm{g} \mu^{-1}$ tRNA, $10 \mathrm{U} \mathrm{ml}^{-1}$ RNase 3 inhibitor). Alexa 594-labelled oligo-(dT) $)_{30}\left(2 \mathrm{ng} \mathrm{l}^{-1}\right.$ in hybridization solution; Invitrogen, USA) 4 was hybridized overnight at room temperature. Slides were then washed for 10 min, once with $550 \mu \mathrm{l}$ of $4 \mathrm{X}$ SSC with $35 \%$ formamide followed by one wash each with $2 \mathrm{X}$ SSC and $1 \mathrm{X}$ SSC. 6 Labelled cells were mounted with 4',6-diamino-2-phenylindole dihydrochloride (DAPI) 7 fluoromount-G (Southern Biotech, Birmingham, AL) for visualization. Images were taken with a 8 fluorescent microscope Zeiss Axioimager M2 stand equipped with a rear-mounted excitation filter 9 wheel. mRNA FISH data were quantified using ImageJ (NIH) software as described previously 10 (Chatterjee et al., 2017). Six cells (three from each replicate) were randomly selected and their 11 fluorescence intensities were measured using Image $J$ with a plot profile analysis of the various 12 points at a regular interval along a line drawn across the nucleus with overhangs covering the 13 cytosol (Hegedüsová et al., 2019). The results are expressed as the average value of relative 14 fluorescence intensity \pm S.D.

\section{$16 \quad 4.8$ Denaturing gel electrophoresis and Northern hybridization}

17 Total RNA was isolated using Trizol reagent and phenol chloroform extraction method as 18 described previously (Lott et al., 2015). Boronate affinity electrophoresis was performed as 19 described previously (Kessler et al., 2018). In brief, $5 \mu \mathrm{g}$ of RNA was resolved by denaturing gel 20 electrophoresis ( $8 \%$ acrylamide, $7 \mathrm{M}$ urea), electroblotted to Zeta probe ${ }^{\circledR}$ (Bio-Rad) membranes, 21 and UV cross-linked $(1200 \mathrm{uJ} \times 100)$. Oxidation control RNA was deacylated and treated with 22 sodium periodate and then the reaction was quenched by addition of $2.5 \mathrm{mM}$ glucose. The 23 membranes were probed with oligonucleotides radiolabeled with $\gamma^{32}$ P-dATP (Supplementary 24 Table S1). Northern hybridization was performed according to the manufacturer's instructions 25 (Bio-Rad). Subsequently, the membranes were exposed overnight to a Phosphorimager screen and 26 analyzed using Typhoon ${ }^{\mathrm{TM}} 9410$ scanner and Image Quant TL software (GE Healthcare) 27 (Hegedüsová et al., 2019). 
1 CLIP was carried out with minor modifications of a previously published method (Mugo \& Erben, 2 2020). Briefly, procyclic form cells $\left(1 \times 10^{7}\right.$ cells $\left.\mathrm{ml}^{-1}\right)$ were harvested and washed once with cold 3 1X PBS buffer ( $\mathrm{pH} 7.4$ ). Cells were resuspended in $25 \mathrm{ml}$ of SM medium without FBS to a 4 concentration of $\sim 5 \times 10^{9}$ cells ml-1, transferred to a $100 \times 15 \mathrm{~mm}$ Petri dish, placed on ice, and UV 5 irradiated at $400 \mathrm{~mJ} / \mathrm{cm} 2$ in a Stratalinker 1800 (Stratagene). Cells were pelleted, washed with 6 PBS, snap frozen in liquid N2, and stored in $-80^{\circ} \mathrm{C}$ until use. Cells were resuspended in $4 \mathrm{ml}$ of 7 lysis buffer (Tris- $\mathrm{HCl}(\mathrm{pH} 7.5), 150 \mathrm{mM} \mathrm{NaCl}, 0.1 \% \mathrm{NP} 40$, and $1 \%$ Triton $\mathrm{X}-100)$ and then lysed 8 by passing 15-20 times through a 21 gauge needle. Cell lysate was cleared by centrifugation at $918000 \mathrm{rpm}$ for 30 minutes at $4{ }^{\circ} \mathrm{C}$. Supernatant was collected, and the $\mathrm{NaCl}$ concentration adjusted 10 to $150 \mathrm{mM}$. Crosslinked DRBD18-RNA complexes were immunopurified from cellular extracts 11 using anti-DRBD18 antibodies (Lott et al., 2015) attached to Protein A fast flow beads (GE 12 Healthcare); anti-Ty1 antibody attached to Protein A fast flow beads served as the control. 13 Captured protein-RNA complexes were washed with wash buffer (Tris-HCl (pH 7.5), 150mM $14 \mathrm{NaCl}, 0.1 \% \mathrm{NP} 40$ ), and $5 \%$ of the beads were taken from each sample and used for Western blot 15 was performed to confirm the pulldown of DRBD18. Beads were treated with DNase 1 (Sigma)

16 followed by proteinase K (Roche). RNA was extracted with phenol/chloroform, and cDNA was 17 prepared using gene specific primers (Table S1) and qRT-PCR was performed as described above. 18 Fold change was calculated as described previously (McAdams et al., 2018). 


\section{FIGURE LEGENDS}

Figure 1. DRBD18 interacts with the general export receptor proteins Mex67 and Mtr2 in 4 vivo.

5 (A) Immunoprecipitations (IP) of T. brucei cells expressing 3XHA-tagged Mtr2 from its genomic locus were carried out using anti-HA beads or IgG Sepharose beads (Control). Bound proteins were eluted using $100 \mathrm{mM}$ glycine and analyzed by Western blot with anti-HA, anti-DRBD18, anti-Mex67, anti-Histone H3 (H3), and anti-EF1 $\alpha$ antibodies. Migration of molecular weight standards $(\mathrm{kDa})$ is indicated on the left. Two biological replicate experiments, each with two technical replicates, were performed, and a representative experiment is shown. (B) HA-Mtr2 and associated proteins were immunoprecipitated from T. brucei cell extracts that were treated with either an RNase cocktail (+RNase) or RNase inhibitor (-RNase). Proteins were eluted and Western blots performed as in (A). Two biological replicate experiments, each with two technical

14 replicates, were performed, and a representative experiment is shown. (C) Quantification of band 15 intensities of the experiment shown in (B). Protein levels were normalized to amount of HA-Mtr2 16 for a given IP. The normalized protein levels from the +RNase IP were then compared to that of 17 the -RNase IP (which was set to 1) to calculate the protein associated with HA-Mtr2. Bar graphs represent the average and standard deviation (SD) of two biological replicates, each with two technical replicates. (D) DRBD18 was immunoprecipitated from HA-Mtr2 expressing cells with

20 specific antibodies. Proteins were eluted and Western blots with anti-HA and anti-Mex67

21 antibodies were performed as in (A). Two biological replicate experiments, each with two

22 technical replicates, were performed, and a representative experiment is shown. (E) Quantification

23 of band intensities from the experiment shown in (D). Bar graphs represent the average and 24 standard deviation (SD) of two biological replicates, each with two technical replicates. 25 Significance was determined by unpaired t-test with Welch's correction. * indicates $\mathrm{p}<0.05$.

Figure 2. In vitro interaction of DRBD18 with Mex67 and Mtr2.

(A) Recombinant His-tagged Mtr2, GST-tagged DRBD18, and GST were expressed, purified by 29 affinity chromatography, and analyzed by $12.5 \%$ SDS-PAGE followed by Coomassie brilliant blue 30 staining. Migration of molecular weight standards $(\mathrm{kDa})$ is indicated on the left. (B) Recombinant 31 GST-DRBD18 or GST were immobilized on glutathione-Sepharose 4B beads, followed by 
1 incubation with His-Mtr2 for GST pulldown assays. Western blot analyses were performed using 2 anti-His and anti-GST antibodies to detect His-Mtr2 and GST or GST-DRBD18, respectively.

3 Purified His-Mtr2 (in lane 1), and GST-DRBD18 or GST on glutathione-Sepharose 4B beads

4 incubated without His-Mtr2 were used as inputs for the experiments (in lane 2 and 3 respectively).

5 Two biological replicates, each with two technical replicates, were performed, and a representative

6 experiment is shown.

8 Figure 3. DRBD18 RNAi does not impact tRNA export.

9 (A) T. brucei cells harboring the DRBD18 RNAi construct were grown for $23 \mathrm{~h}$ in the presence 10 (+Dox) or absence (-Dox) of doxycycline. Total RNA was separated by boronate affinity 11 electrophoresis followed by Northern blotting. A probe recognizing the tRNA ${ }^{\text {Tyr }} 3$ ' exon was used 12 to detect queuosine (Q)-modified tRNA ${ }^{\text {Tyr }}$ levels, based on the electrophoretic shift caused by the 13 presence of Q as described elsewhere (Igloi \& Kössel, 1985). G indicates unmodified tRNA ${ }^{\text {Tyr. }}$ 14 The levels of Q-modified tRNA are shown below the panel. WT and OX indicate untreated and 15 sodium periodate treated cells as a negative control. A probe against tRNA ${ }^{\text {Glu }}$ (middle panel) and 16 the ethidium bromide stained gel (bottom panel) were used as a loading controls. (B and C) 17 Subcellular localization of tRNA ${ }^{\mathrm{Tyr}}$ (red-Cy3) (B) and tRNA ${ }^{\mathrm{Glu}}$ (green-AF488) (C) in uninduced 18 (-Dox) or induced (+Dox) DRBD18 RNAi cells were monitored by fluorescence in 19 situ hybridization (FISH). DAPI was used to stain the DNA of the nucleus (N) and the kinetoplast 20 (K). An overlay of the FISH and DAPI signals (Merge) is shown.

Figure 4. DRBD18 RNAi causes partial accumulation of mRNA in the nucleus.

23 PF T. brucei cells harboring the DRBD18 RNAi construct were used to assess the relative 24 abundance of mRNA in the nucleus upon depletion of DRBD18. (A) Subcellular localization of 25 total mRNA in uninduced (-Dox) or induced (+Dox) DRBD18 RNAi cells was monitored by 26 fluorescence in situ hybridization (FISH) using fluorescently labelled oligo(dT). DAPI was used 27 to stain the DNA of the nucleus $(\mathrm{N})$ and the kinetoplast $(\mathrm{K})$. An overlay of the FISH and DAPI 28 signals (Merge) is shown. Bars, $3.2 \mu \mathrm{m}$. Images of individual cells were enlarged to enhance 29 visualization of the nucleus. (B) Quantification of the fluorescence intensities of FISH (red-Alexa 30 594) and DAPI (blue) fluorophores were calculated on a line drawn across the nucleus, with 31 overhangs covering the cytosol, using ImageJ (NIH) software. Two biological replicate 
1 experiments were performed. The relative intensities represent the average \pm SD from six 2 randomly selected cells (three from each replicate).

Figure 5. DRBD18 RNAi inhibits export of a subset of mRNAs from nucleus to cytosol and associates with differentially transported mRNAs.

(A) Volcano plot (generated from RNAseq data) of transcripts whose abundance is decreased in cytosol but unchanged in whole cell RNA (corrected p-value <0.05) upon DRBD18 RNAi.

8 Transcripts with fold change $(\mathrm{FC})>1.5$ are colored blue, and those with $\mathrm{FC}<1.5$ are red.

(B) Representative transcripts, identified by the last four to seven digits of their TriTrypDB numbers, were quantified

11 by qRT-PCR in whole cell and cytosolic fractions. Relative abundance represents RNA levels in induced (+Dox) cells compared to levels in uninduced (-Dox) cells. RNA levels were normalized

13 to $18 \mathrm{~S}$ rRNA. Values represent the mean of three biological replicates, each with three technical

14 replicates. Significance was determined by unpaired t-test. ${ }^{*} \mathrm{p}<0.01 ;{ }^{*} \mathrm{p}<0.05$. (C) Enrichment

15 of transcripts shown in (B) in anti-DRBD18 immunoprecipitations relative to a non-specific

16 antibody control was measured by UV Cross-Linking Immunoprecipitation (CLIP). The 7.7400

17 transcript serves as a negative control. Values represent the mean of three biological replicates,

18 each with three qRT-PCR technical replicates. A value of 1 (dotted line) indicates no enrichment.

Figure 6. DRBD18 RNAi causes partial accumulation of export receptors in the nucleus.

21 (A) The relative abundance of Mtr2 and Mex67 in nuclear and cytosolic fractions in uninduced (-

22 Dox) or induced (+Dox) DRBD18 RNAi cells were analyzed by subcellular fractionation followed

23 by Western blot analysis. Anti-Histone H3 (H3) and anti-EF1 $\alpha$ antibodies were used as loading

24 controls for nuclear and cytosolic fractions, respectively. (B) Quantification of Western blots in

25 (A). Relative abundance of Mex67 or Mtr2 in nuclear and cytosolic fractions was normalized to

26 the expression of Histone $\mathrm{H} 3$ and $\mathrm{EF} 1 \alpha$, respectively. The normalized protein expression in

27 nuclear and cytosolic fractions from the +Dox were then compared to that of the -Dox (which was

28 set to 1) to calculate the relative abundances in the DRBD18 knockdown. Bar graphs represent the 29 average and standard deviation (SD) of four samples (two biological replicates, each with two 30 technical replicates). Significance was determined by unpaired t-test with Welch's correction. *p $31<0.05$ and $\mathrm{ns}=$ non-significant. 
2 Figure 7. Model of the role of DRBD18 in mRNA export. A model summarizing the potential

3 role of DRBD18 in mRNA export from nucleus to cytosol through the Nuclear Pore Complex

4 (NPC; see text for details). Inset shows the detailed architecture of NPC and its structural

5 components (Nups). Core scaffold Nups, dark grey (outer ring) and light grey (inner ring); FG

6 Nups, green; nuclear basket, black; cytosolic filament, blue. The NPC has left to right symmetry,

7 and specific features are labeled on only one side for clarity, although they are present on both

8 sides. DRBD18, orange; Mtr2, yellow; Mex67, olive green. DRBD18 may promote association of

9 select mRNAs with Mex67/Mtr2 and likely acts as a chaperone that facilitates passage of the

10 Mex67/Mtr2-DRBD18 mRNP across the NPC due to its interactions with the outer ring and Nup76

11 complex.

12 


\section{AUTHOR CONTRIBUTIONS}

2

13 Virulence of Parasites [CZ.02.1.01/0.0/0.0/16_019/0000759 to ZP]. 


\section{REFERENCES}

Andrews, S., (2010) FastQC: a quality control tool for high throughput sequence data. In.: Babraham Bioinformatics, Babraham Institute, Cambridge, United Kingdom.

Aphasizheva, I., Alfonzo, J., Carnes, J., Cestari, I., Cruz-Reyes, J., Göringer, H.U., Hajduk, S., Lukeš, J., Madison-Antenucci, S., Maslov, D.A., McDermott, S.M., Ochsenreiter, T., Read, L.K., Salavati, R., Schnaufer, A., Schneider, A., Simpson, L., Stuart, K., Yurchenko, V., Zhou, Z.H., Zíková, A., Zhang, L., Zimmer, S., and Aphasizhev, R. (2020) Lexis and grammar of mitochondrial RNA processing in trypanosomes. Trends Parasitol 36: 337-355.

Aslett, M., Aurrecoechea, C., Berriman, M., Brestelli, J., Brunk, B.P., Carrington, M., Depledge, D.P., Fischer, S., Gajria, B., and Gao, X. (2010) TriTrypDB: a functional genomic resource for the Trypanosomatidae. Nucl Acids Res 38: D457-D462.

Bangs, J.D. (2018) Evolution of antigenic variation in African trypanosomes: Variant surface glycoprotein expression, structure, and function. Bioessays 40: e1800181.

Braun, I.C., Herold, A., Rode, M., and Izaurralde, E. (2002) Nuclear export of mRNA by TAP/NXF1 requires two nucleoporin-binding sites but not p15. Mol Cell Biol 22: 54055418.

Brugiolo, M., Botti, V., Liu, N., Müller-McNicoll, M., and Neugebauer, K.M. (2017) Fractionation iCLIP detects persistent SR protein binding to conserved, retained introns in chromatin, nucleoplasm and cytoplasm. Nucl Acids Res 45: 10452-10465.

Büscher, P., Cecchi, G., Jamonneau, V., and Priotto, G. (2017) Human African trypanosomiasis. Lancet 390: 2397-2409.

Chatterjee, K., Majumder, S., Wan, Y., Shah, V., Wu, J., Huang, H.-Y., and Hopper, A.K. (2017) Sharing the load: Mex67-Mtr2 cofunctions with Los1 in primary tRNA nuclear export. Genes Devel 31: 2186-2198.

Clayton, C. (2019) Regulation of gene expression in trypanosomatids: living with polycistronic transcription. Open Biol 9: 190072.

Dean, S., Sunter, J., Wheeler, R.J., Hodkinson, I., Gluenz, E., and Gull, K. (2015) A toolkit enabling efficient, scalable and reproducible gene tagging in trypanosomatids. Open Biol 5: 140197-140197.

Dobin, A., Davis, C.A., Schlesinger, F., Drenkow, J., Zaleski, C., Jha, S., Batut, P., Chaisson, M., and Gingeras, T.R. (2013) STAR: ultrafast universal RNA-seq aligner.

Bioinformatics 29: 15-21.

Dostalova, A., Käser, S., Cristodero, M., and Schimanski, B. (2013) The nuclear mRNA export receptor Mex67-Mtr2 of Trypanosoma brucei contains a unique and essential zinc finger motif. Mol Microbiol 88: 728-739.

Droll, D., Minia, I., Fadda, A., Singh, A., Stewart, M., Queiroz, R., and Clayton, C. (2013) Posttranscriptional regulation of the trypanosome heat shock response by a zinc finger protein. PLoS Pathog 9: e1003286.

Ewels, P., Magnusson, M., Lundin, S., and Käller, M. (2016) MultiQC: summarize analysis results for multiple tools and samples in a single report. Bioinformatics 32: 3047-3048.

Goos, C., Dejung, M., Wehman, A.M., M-Natus, E., Schmidt, J., Sunter, J., Engstler, M., Butter, F., and Kramer, S. (2018) Trypanosomes can initiate nuclear export co-transcriptionally. Nucl Acids Res 47: 266-282. 
Hayman, M.L., Miller, M.M., Chandler, D.M., Goulah, C.C., and Read, L.K. (2001) The trypanosome homolog of human p32 interacts with RBP16 and stimulates its gRNA binding activity. Nucl Acids Res 29: 5216-5225.

Hegedűsová, E., Kulkarni, S., Burgman, B., Alfonzo, J.D., and Paris, Z. (2019) The general mRNA exporters Mex67 and Mtr2 play distinct roles in nuclear export of tRNAs in Trypanosoma brucei. Nucl Acids Res 47: 8620-8631.

Howard, J.M., and Sanford, J.R. (2015) The RNAissance family: SR proteins as multifaceted regulators of gene expression. Wiley Interdiscip Rev RNA 6: 93-110.

Igloi, G.L., and Kössel, H. (1985) Affinity electrophoresis for monitoring terminal phosphorylation and the presence of queuosine in RNA. Application of polyacrylamide containing a covalently bound boronic acid. Nucl Acids Res 13: 6881-6898.

Jensen, R.E., and Englund, P.T. (2012) Network news: the replication of kinetoplast DNA. Annu Rev Microbiol 66: 473-491.

Kafková, L., Debler, E.W., Fisk, J.C., Jain, K., Clarke, S.G., and Read, L.K. (2017) The major protein arginine methyltransferase in Trypanosoma brucei functions as an enzyme-p complex. J Biol Chem 292: 2089-2100.

Keating, J., Yukich, J.O., Sutherland, C.S., Woods, G., and Tediosi, F. (2015) Human African trypanosomiasis prevention, treatment and control costs: A systematic review. Acta Tropica 150: 4-13.

Kessler, A.C., Kulkarni, S.S., Paulines, M.J., Rubio, M.A.T., Limbach, P.A., Paris, Z., and Alfonzo, J.D. (2018) Retrograde nuclear transport from the cytoplasm is required for tRNA(Tyr) maturation in T. brucei. RNA Biol 15: 528-536.

Klebanov-Akopyan, O., Mishra, A., Glousker, G., Tzfati, Y., and Shlomai, J. (2018) Trypanosoma brucei UMSBP2 is a single-stranded telomeric DNA binding protein essential for chromosome end protection. Nucl Acids Res 46: 7757-7771.

Klein, C., Terrao, M., Inchaustegui Gil, D., and Clayton, C. (2015) Polysomes of Trypanosoma brucei: Association with initiation factors and RNA-binding proteins. PLoS One 10: e0135973.

Köhler, A., and Hurt, E. (2007) Exporting RNA from the nucleus to the cytoplasm. Nat Rev Mol Cell Biol 8: 761-773.

Kolev, N.G., Ramey-Butler, K., Cross, G.A., Ullu, E., and Tschudi, C. (2012) Developmental progression to infectivity in Trypanosoma brucei triggered by an RNA-binding protein. Science 338: 1352-1353.

Kolev, N.G., Ullu, E., and Tschudi, C. (2014) The emerging role of RNA-binding proteins in the life cycle of Trypanosoma brucei. Cell Microbiol 16: 482-489.

Kopylova, E., Noé, L., and Touzet, H. (2012) SortMeRNA: fast and accurate filtering of ribosomal RNAs in metatranscriptomic data. Bioinformatics 28: 3211-3217.

Krueger, F. (2015) Trim galore. https://github.com/FelixKrueger/TrimGalore

Li, B., and Dewey, C.N. (2011) RSEM: accurate transcript quantification from RNA-Seq data with or without a reference genome. BMC Bioinformat 12: 323.

Liu, B., Kamanyi Marucha, K., and Clayton, C. (2020) The zinc finger proteins ZC3H20 and ZC3H21 stabilise mRNAs encoding membrane proteins and mitochondrial proteins in insect-form Trypanosoma brucei. Mol Microbiol 113: 430-451.

Lott, K., Mukhopadhyay, S., Li, J., Wang, J., Yao, J., Sun, Y., Qu, J., and Read, L.K. (2015) Arginine methylation of DRBD18 differentially impacts its opposing effects on the trypanosome transcriptome. Nucl Acids Res 43: 5501-5523. 
Love, M.I., Huber, W., and Anders, S. (2014) Moderated estimation of fold change and dispersion for RNA-seq data with DESeq2. Genome Biol 15: 550.

Martin, M. (2011) Cutadapt removes adapter sequences from high-throughput sequencing reads. EMBnet. journal 17: 10-12.

Maslon, M.M., Heras, S.R., Bellora, N., Eyras, E., and Cáceres, J.F. (2014) The translational landscape of the splicing factor SRSF1 and its role in mitosis. Elife 3: e02028.

Matthews, K.R. (2005) The developmental cell biology of Trypanosoma brucei. J Cell Sci 118: 283-290.

McAdams, N.M., Simpson, R.M., Chen, R., Sun, Y., and Read, L.K. (2018) MRB7260 is essential for productive protein-RNA interactions within the RNA editing substrate binding complex during trypanosome RNA editing. RNA 24: 540-556.

Meola, N., and Jensen, T.H. (2017) Targeting the nuclear RNA exosome: Poly(A) binding proteins enter the stage. RNA Biol 14: 820-826.

Michaeli, S. (2011) Trans-splicing in trypanosomes: machinery and its impact on the parasite transcriptome. Future Microbiol 6: 459-474.

Mugo, E., and Clayton, C. (2017) Expression of the RNA-binding protein RBP10 promotes the bloodstream-form differentiation state in Trypanosoma brucei. PLoS Pathog 13: e1006560.

Mugo, E., and Erben, E.D. (2020) Identifying trypanosome protein-RNA interactions using RIPSeq. Methods Mol Biol 2116: 285-294.

Müller-McNicoll, M., Botti, V., de Jesus Domingues, A.M., Brandl, H., Schwich, O.D., Steiner, M.C., Curk, T., Poser, I., Zarnack, K., and Neugebauer, K.M. (2016) SR proteins are NXF1 adaptors that link alternative RNA processing to mRNA export. Genes Dev 30: 553-566.

Obado, S.O., Brillantes, M., Uryu, K., Zhang, W., Ketaren, N.E., Chait, B.T., Field, M.C., and Rout, M.P. (2016) Interactome mapping reveals the evolutionary history of the nuclear pore complex. PLOS Biology 14: e1002365.

Rehwinkel, J., Herold, A., Gari, K., Köcher, T., Rode, M., Ciccarelli, F.L., Wilm, M., and Izaurralde, E. (2004) Genome-wide analysis of mRNAs regulated by the THO complex in Drosophila melanogaster. Nat Struct Mol Biol 11: 558-566.

Rico, E., Ivens, A., Glover, L., Horn, D., and Matthews, K.R. (2017) Genome-wide RNAi selection identifies a regulator of transmission stage-enriched gene families and cell-type differentiation in Trypanosoma brucei. PLoS Pathog 13: e1006279.

Rink, C., and Williams, N. (2019) Unique interactions of the nuclear export receptors TbMex67 and TbMtr2 with components of the 5S ribonuclear particle in Trypanosoma brucei. mSphere 4: e00471-19.

Schwede, A., Manful, T., Jha, B.A., Helbig, C., Bercovich, N., Stewart, M., and Clayton, C. (2009) The role of deadenylation in the degradation of unstable mRNAs in trypanosomes. Nucl Acids Res 37: 5511-5528.

Scott, D.D., Aguilar, L.C., Kramar, M., and Oeffinger, M. (2019) It's not the destination, it's the journey: Heterogeneity in mRNA export mechanisms. Adv Exp Med Biol 1203: 33-81.

Serpeloni, M., Moraes, C.B., Muniz, J.R., Motta, M.C., Ramos, A.S., Kessler, R.L., Inoue, A.H., daRocha, W.D., Yamada-Ogatta, S.F., Fragoso, S.P., Goldenberg, S., Freitas-Junior, L.H., and Avila, A.R. (2011a) An essential nuclear protein in trypanosomes is a component of mRNA transcription/export pathway. PLoS One 6: e20730. 
Serpeloni, M., Vidal, N.M., Goldenberg, S., Avila, A.R., and Hoffmann, F.G. (2011b) Comparative genomics of proteins involved in RNA nucleocytoplasmic export. $B M C$ Evol Biol 11: 7.

Strambio-De-Castillia, C., Niepel, M., and Rout, M.P. (2010) The nuclear pore complex: bridging nuclear transport and gene regulation. Nat Rev Mol Cell Biol 11: 490-501.

Tetenbaum-Novatt, J., and Rout, M.P. (2010) The mechanism of nucleocytoplasmic transport through the nuclear pore complex. Cold Spring Harb Symp Quant Biol 75: 567-584.

Tudek, A., Schmid, M., and Jensen, T.H. (2019) Escaping nuclear decay: the significance of mRNA export for gene expression. Curr Genet 65: 473-476.

Tudek, A., Schmid, M., Makaras, M., Barrass, J.D., Beggs, J.D., and Jensen, T.H. (2018) A nuclear export block triggers the decay of newly synthesized polyadenylated RNA. Cell Rep 24: 2457-2467.e2457.

Tutucci, E., and Stutz, F. (2011) Keeping mRNPs in check during assembly and nuclear export. Nature Rev Mol Cell Biol 12: 377-384.

Verner, Z., Basu, S., Benz, C., Dixit, S., Dobáková, E., Faktorová, D., Hashimi, H., Horáková, E., Huang, Z., Paris, Z., Peña-Diaz, P., Ridlon, L., Týč, J., Wildridge, D., Zíková, A., and Lukeš, J. (2015) Malleable mitochondrion of Trypanosoma brucei. Int Rev Cell Mol Biol 315: $73-151$.

Wickramasinghe, V.O., Andrews, R., Ellis, P., Langford, C., Gurdon, J.B., Stewart, M., Venkitaraman, A.R., and Laskey, R.A. (2014) Selective nuclear export of specific classes of mRNA from mammalian nuclei is promoted by GANP. Nucl Acids Res 42: 5059-5071.

Wickramasinghe, V.O., and Laskey, R.A. (2015) Control of mammalian gene expression by selective mRNA export. Nat Rev Mol Cell Biol 16: 431-442.

Zimmer, S.L., Simpson, R.M., and Read, L.K. (2018) High throughput sequencing revolution reveals conserved fundamentals of U-indel editing. Wiley Interdiscip Rev RNA: e1487. 
Figure 1

A

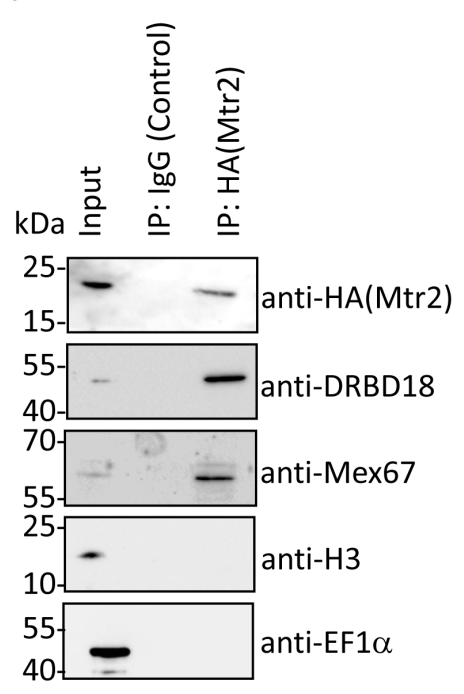

B

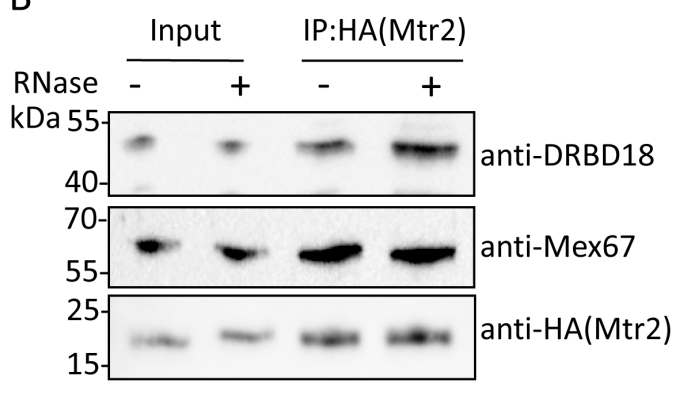

D

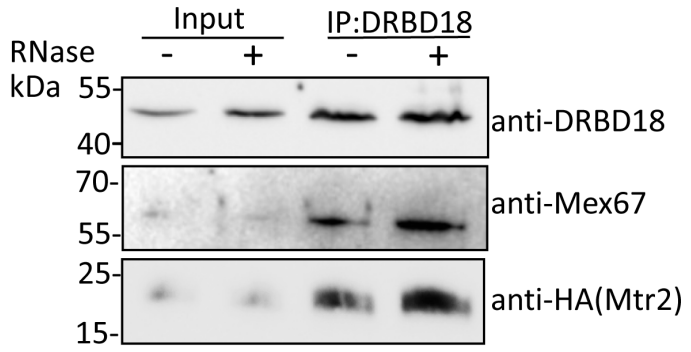

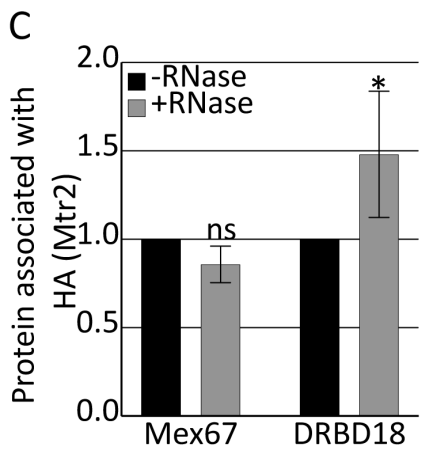

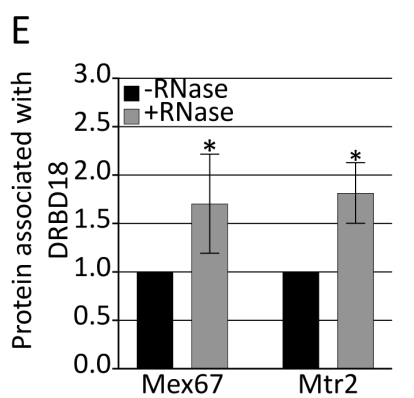




\section{Figure 2}

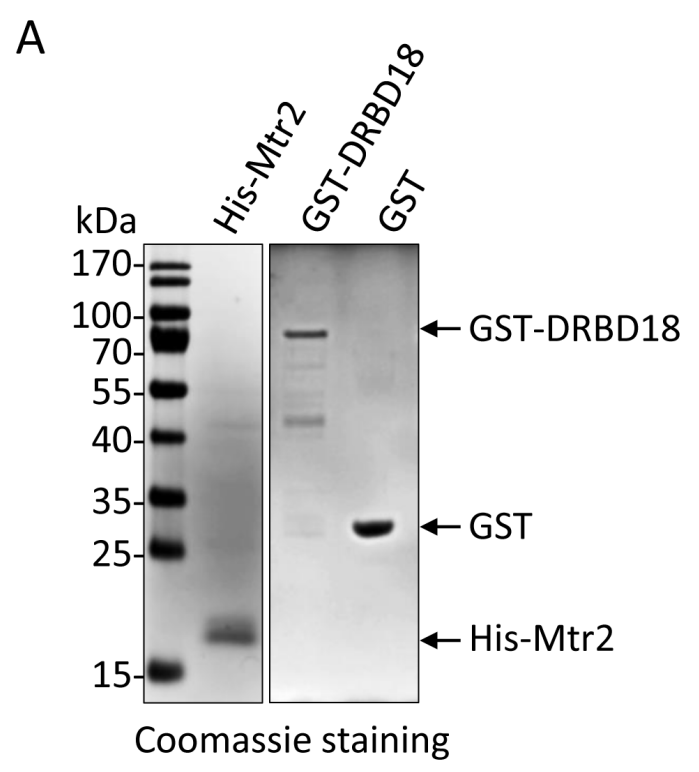

B

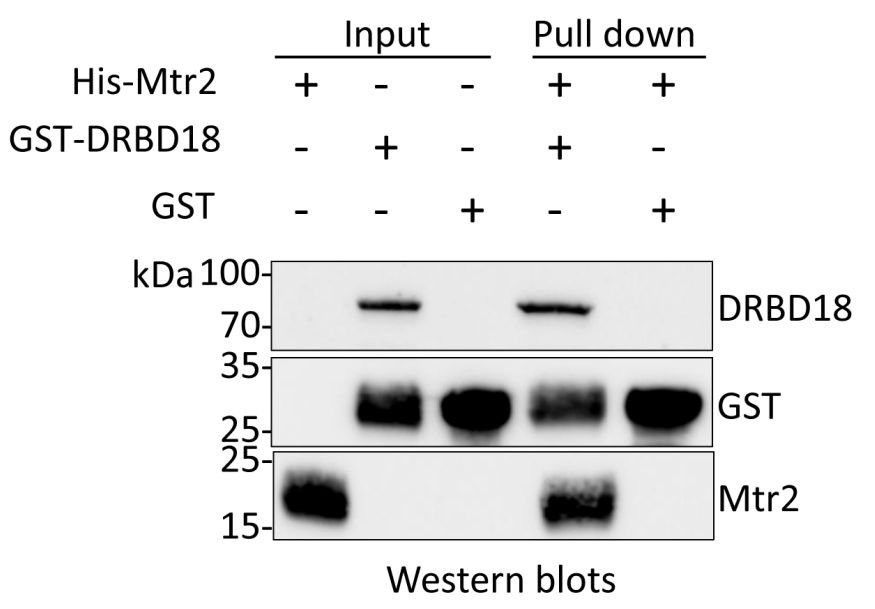


Figure 3
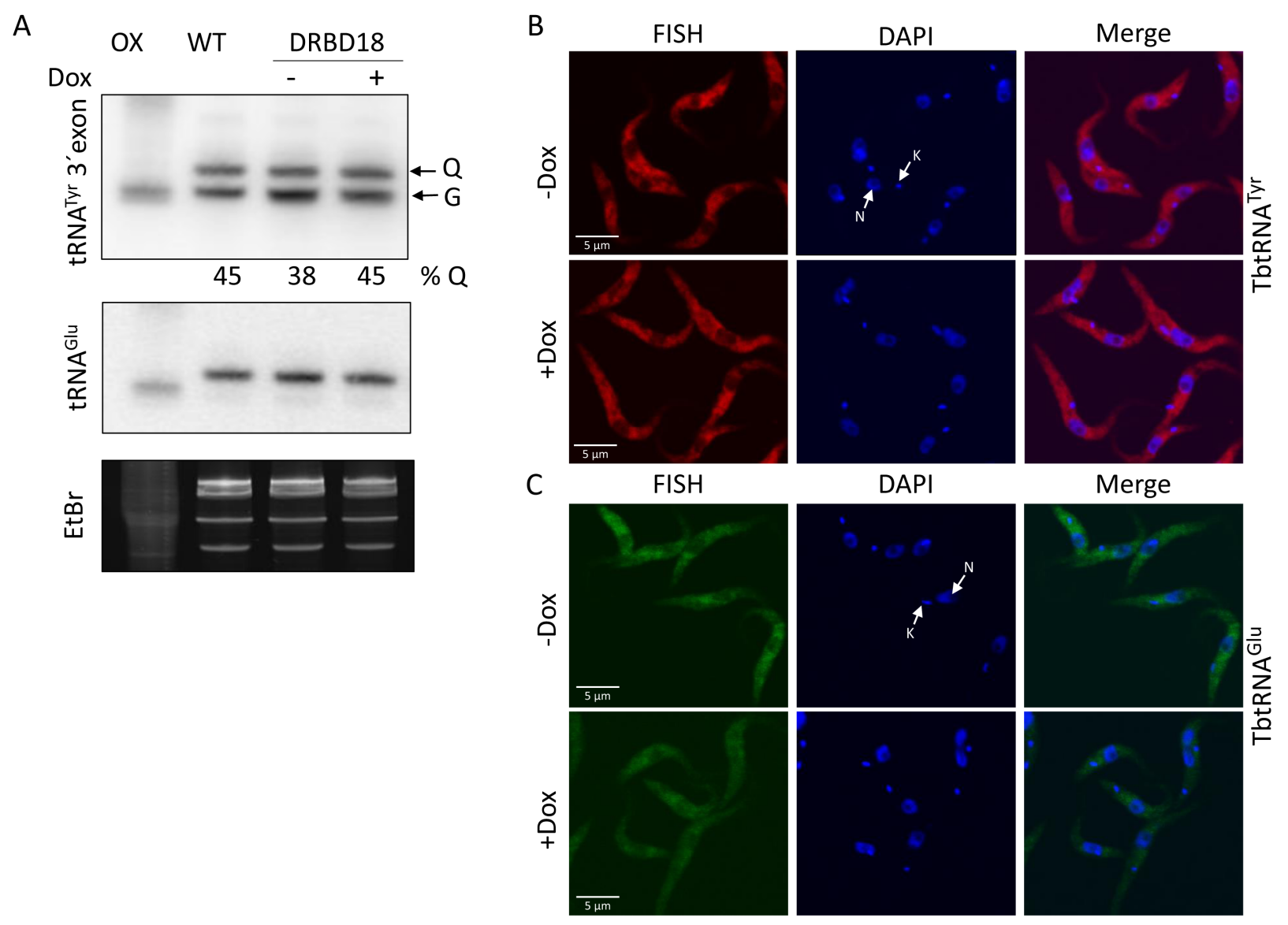
Figure 4

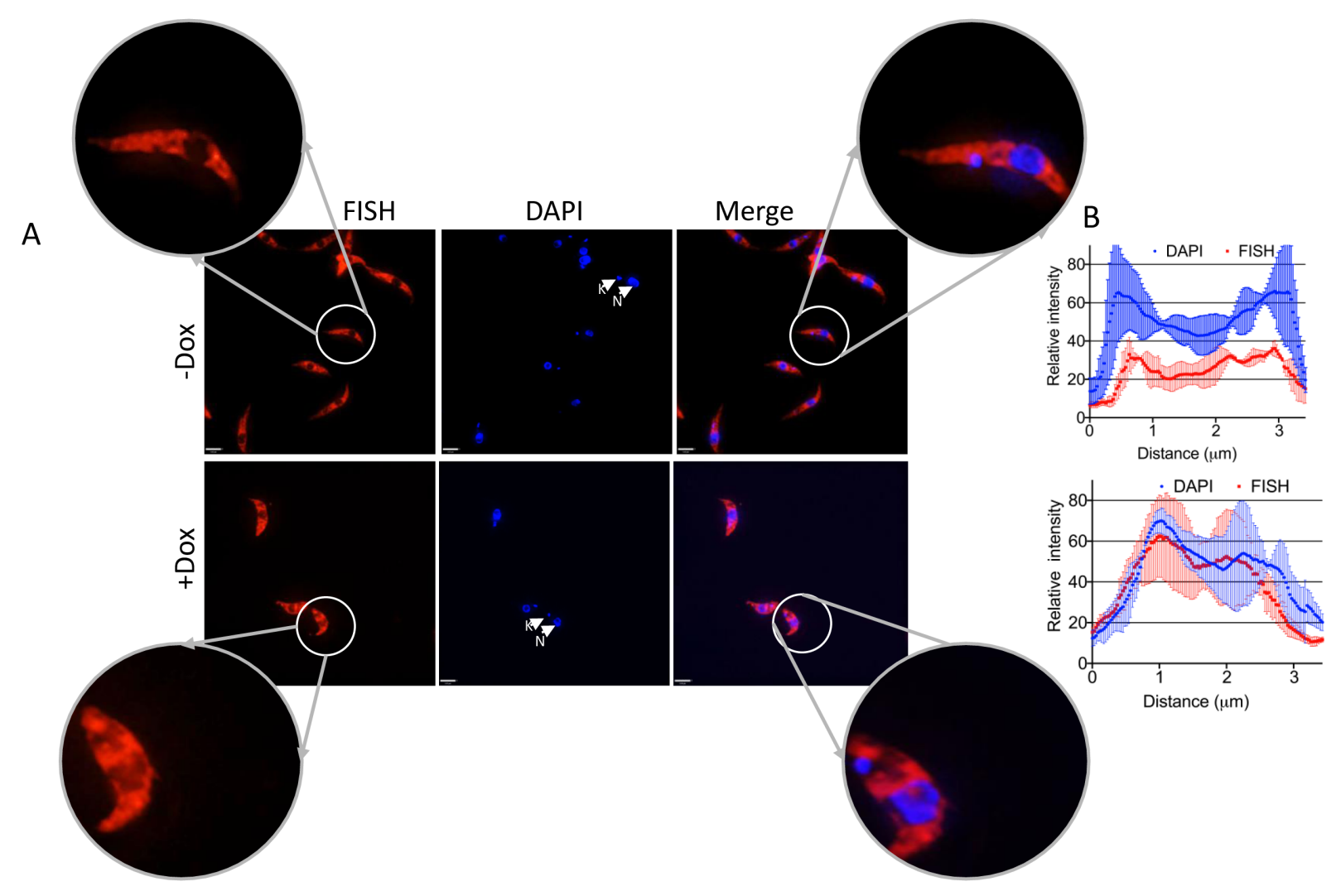


Figure 5

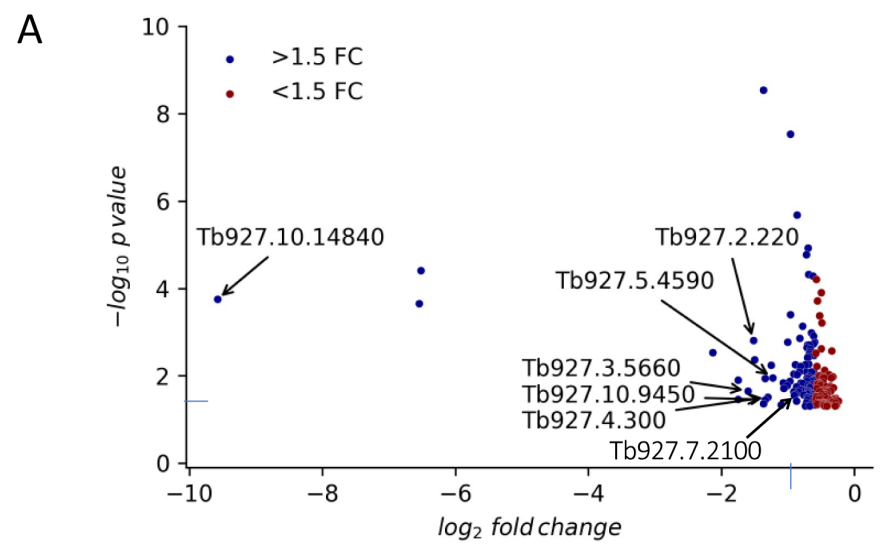

B

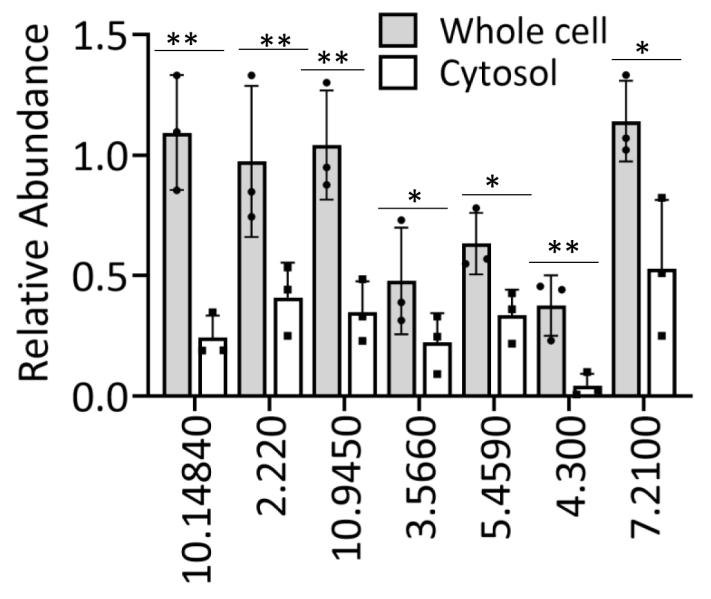

C

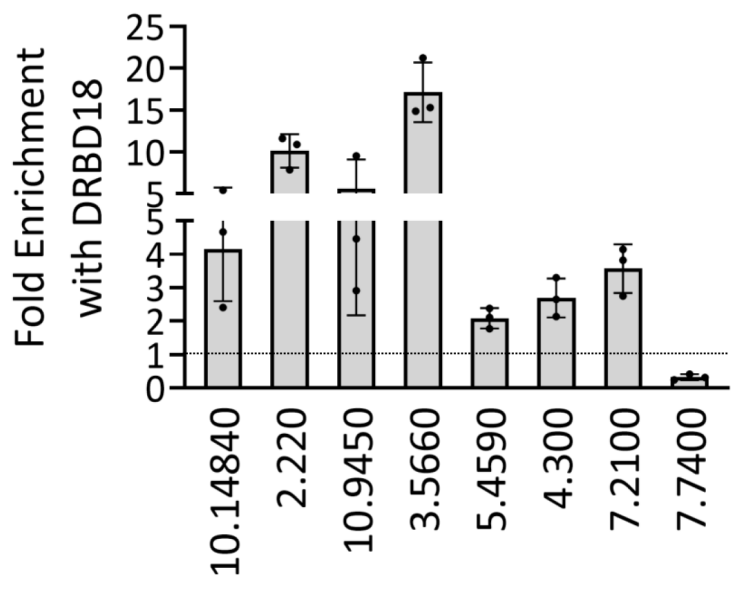




\section{Figure 6}

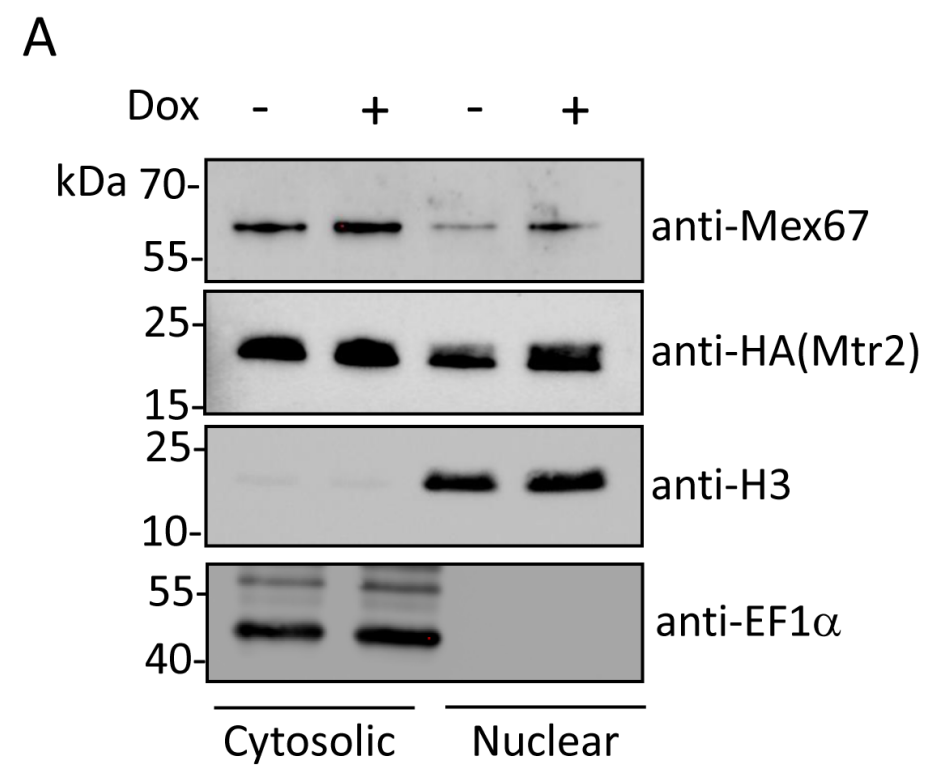

B

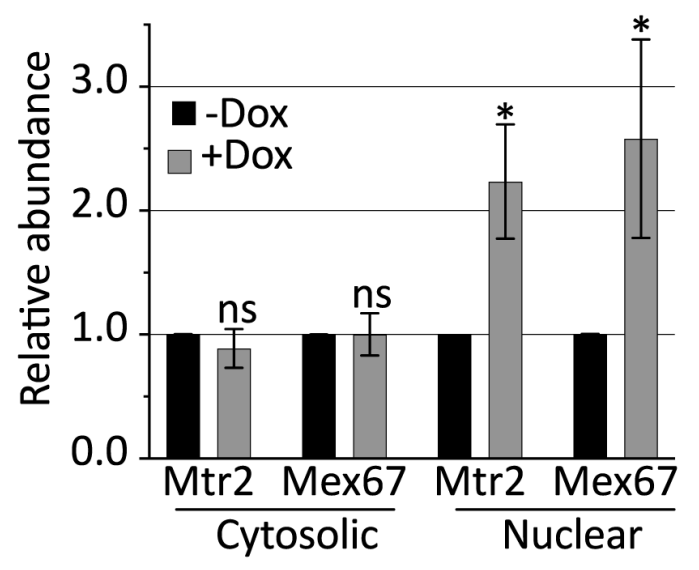




\section{Figure 7}

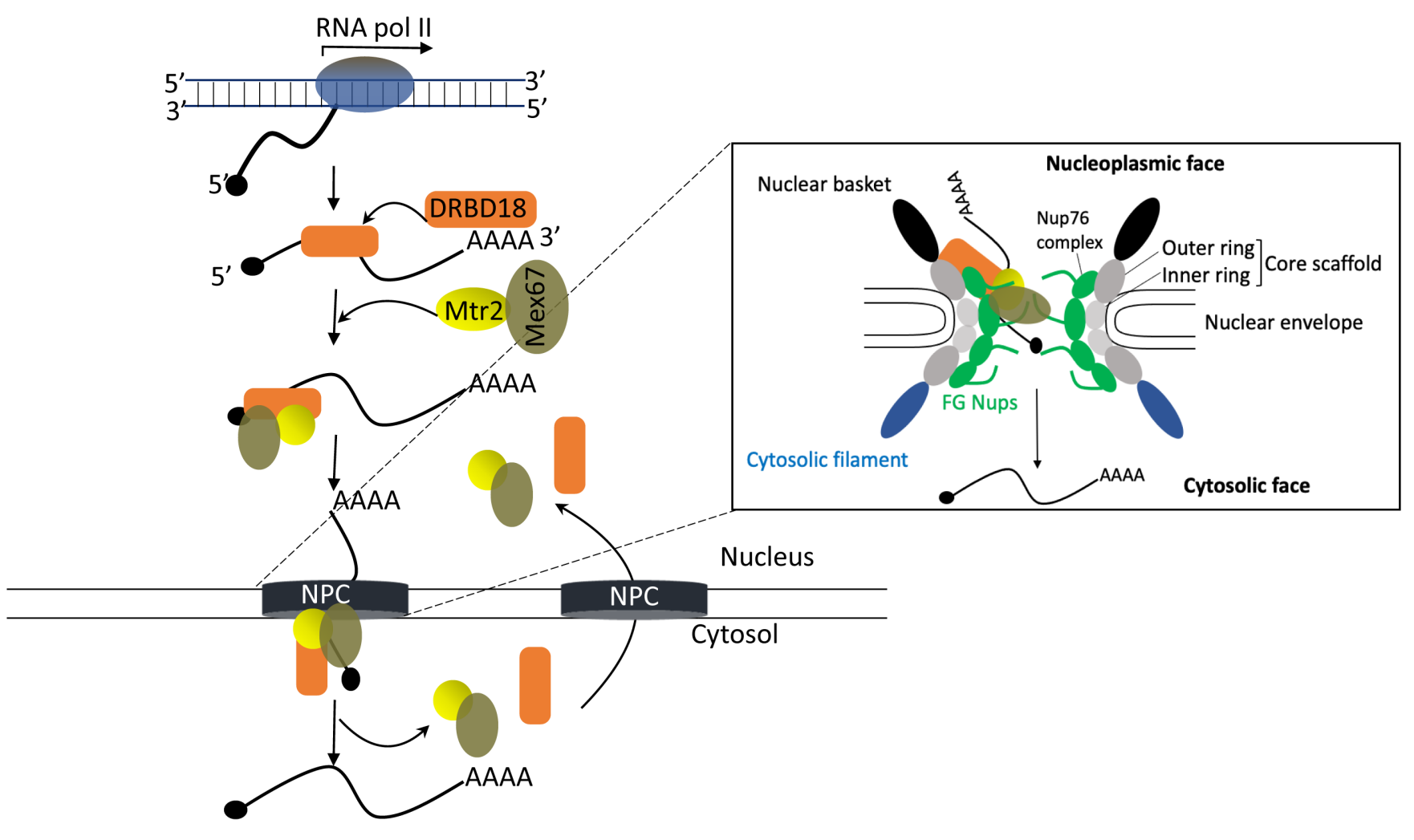

